\title{
FAST SOLAR ELECTRONS, INTERPLANETARY PLASMA AND km-WAVE TYPE-III RADIO BURSTS OBSERVED FROM THE IMP-6 SPACECRAFT
}

\author{
HECTOR ALVAREZ \\ Radio Astronomy Observatory, University of Michigan, Ann Arbor, Mich., U.S.A. \\ ROBERT P. LIN \\ Space Sciences Laboratory, University of California, Berkeley, U.S.A. \\ and \\ SAMUEL J. BAME \\ Los Alamos Scientific Laboratory, U.S.A.
}

(Received 15 April, 1975)

\begin{abstract}
IMP-6 spacecraft observations of low frequency radio emission, fast electrons, and solar wind plasma are used to examine the dynamics of the fast electron streams which generate solar typeIII radio bursts. Of twenty solar electron events observed between April, 1971 and August, 1972, four were found to be amenable to detailed analysis. Observations of the direction of arrival of the radio emission at different frequencies were combined with the solar wind density and velocity measurements at $1 \mathrm{AU}$ to define an Archimedean spiral trajectory for the radio burst exciter. The propagation characteristics of the exciter and of the fast electrons observed at $1 \mathrm{AU}$ were then conpared. We find that: (1) the fast electrons excite the radio emission at the second harmonic; (2) the total distance travelled by the electrons was between 30 and $70 \%$ longer than the length of the smooth spiral defined by the radio observations; (3) this additional distance travelled is the result of scattering of the electrons in the interplanetary medium; (4) the observations are consistent with negligible true energy loss by the fast electrons.
\end{abstract}

\section{Introduction}

Solar type-III radio bursts are generated by a stream of fast particles ejected from the Sun (Wild, 1950). The particles presumably generate plasma waves at frequencies near the local plasma frequency, these plasma waves then scatter off ion density inhomogeneities and other plasma waves to produce the observed electromagnetic radiation at the plasma frequency (fundamental) and twice the plasma frequency (2nd harmonic), respectively (see Smith (1974) for review). As the fast particles go upward in the corona and into the interplanetary medium the radio emission exhibits the rapid drift from high to low frequency which is characteristic of the type-III bursts. In the past few years spacecraft observations of solar radio emission and energetic particle fluxes in the near-Earth interplanetary medium have provided strong evidence that electrons of $\sim 5$ to $10^{2} \mathrm{keV}$ energies excite solar type-III radio bursts (Alvarez et al., 1972; Frank and Gurnett, 1972; Lin et al., 1973).

One important question is how much of the fast particle energy is converted into plasma waves. From spacecraft observations Fainberg et al. (1973) found an apparent 
deceleration of the exciter from the Sun to $1 \mathrm{AU}$, indicative of $\sim 75 \%$ loss in energy. However, when energetic charged particles propagate through the interplanetary medium they are scattered by irregularities in the interplanetary magnetic field (see reviews by Lin (1974) and McCracken and Rao, (1970)). This scattering will increase the particle path length above that for travel along a smooth spiral, thus leading an apparent deceleration of the particles.

The identification of fast solar electrons as type-III burst exciters provides a means of studying the propagation of these electrons and the characteristics of the interplanetary medium, in particular, the fluctuations in the magnetic field. In this paper we examine the dynamics of the fast electron stream using spacecraft observations of low frequency radio emission, fast electrons, and solar wind plasma. These observations enable us to define the trajectory of the burst exciter, determine whether the radiation is emitted at the fundamental or 2 nd harmonic, and compare the exciter's characteristics with those of the fast electrons.

We find that the observations are consistent with the interpretation that the apparent deceleration of the radio source is entirely due to scattering of the electrons in the interplanetary medium.

\section{The Experiments}

The three experiments involved in this study were aboard the Interplanetary Monitoring Platform (IMP-6) Earth satellite. This spacecraft was launched on March 13, 1971 into a highly eccentric orbit with apogee of about $200000 \mathrm{~km}$ and orbital peri od of $\approx 4$ days.

The University of California, Berkeley, experiment detects electrons with energies between 18 and $400 \mathrm{keV}$ with an energy resolution of $\approx 8 \mathrm{keV}$ FWHM. A detailed description is given in Lin et al. (1973).

The solar radio bursts were detected by the University of Michigan radiometer. This instrument has channels at $3.5 \mathrm{MHz}, 900,600,350,230,130,80$ and $50 \mathrm{kHz}$. Each channel is sampled every $5.2 \mathrm{~s}$. Due to the spin stabilization of the spacecraft the antenna output is amplitude modulated. From the phase of this spin modulation the radio astronomy experiment can determine the direction of arrival of the radio waves at the different frequencies. A detailed description of the radiometer is given in Alvarez et al. (1974).

The Los Alamos Experiment measured the density and speed of the solar wind protons. These data are presented here in the form of one-hour averages. The IMP- 6 hemispherical plasma analyzer basically had the same design as analyzers developed and flown in the Vela series (Bame et al., 1967). Specific details of the present experiment and data analysis techniques are given in Feldman et al. (1973).

\section{The Data}

Between April, 1971 and August, 1972 twenty impulsive solar electron events were clearly observed by IMP-6. All of these were accompanied by type-III radio bursts 
observed to low frequencies. For twelve of these events the radio data were free of noise and data gaps. The radio observations for six of these twelve events could be fitted to a trajectory along a simple Archimedean spiral. For four of the six events the electron fluxes during the event onset were free from contamination by terrestrial particle fluxes and large enough to provide good statistical accuracy to be analyzed in detail. These four events, which occurred on 16 May 1971, 28 February 1972, 27 March 1972 and 19 August 1972, are used in the analysis presented here. All four were observed to drift from $3.5 \mathrm{MHz}$ to $50 \mathrm{kHz}$, and the directions of arrival of the radio emission in each could be determined down to $80 \mathrm{kHz}$. Data on $\mathrm{H} \alpha$ flares and radio bursts observed from ground were taken from Solar-Geophysical Data (NOAA).

\section{The Method of Analysis}

We assume that the type-III radio burst exciter propagates outward along a simple Archimedean spiral in the ecliptic plane (see Figure 1). Then the radio source apparent angular extent can be estimated from the depth of the spin modulation of the radio signal. From our preliminary analysis the radio source size appears to be $\gtrsim 40^{\circ}$ as measured from the Sun. In the following discussion the position of the radio source will refer to the centroid of the source.

The shape and location of the spiral are determined by the velocity of the solar wind, $V$, which is assumed to be constant from the Sun to the Earth, and by the helio graphic longitude, $\theta_{F}$, of the foot of the spiral at the Sun. The equation for the spiral is then

$$
\theta(r)=\theta_{F}-\frac{r \Omega}{V}
$$

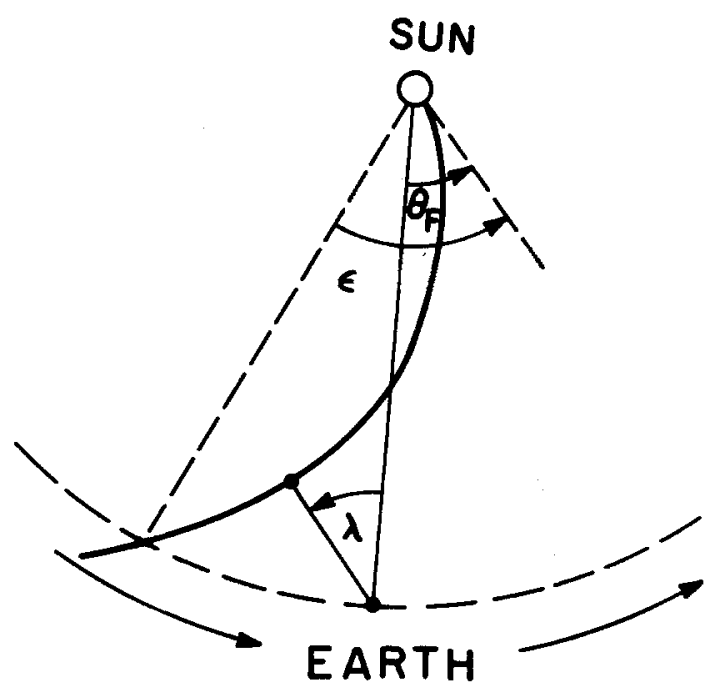

Fig. 1. Model geometry of the type-III burst exciter trajectory viewed from the north. 
or

$$
\theta(r)=\theta_{F}-\frac{\varepsilon}{215} r
$$

where $r$ is heliocentric distance in solar radii $\left(R_{\odot}\right), \Omega=2.7 \times 10^{-6} \mathrm{rad} \mathrm{s}^{-1}$ is the solar rotation rate at the equator relative to the Sun-Earth line and, $\varepsilon=2.3 \times 10^{4} / V \mathrm{deg}$ is the angular displacement of the radius vector between the Sun and $1 \mathrm{AU}\left(215 R_{\odot}\right)$ for $V$ in $\mathrm{km} \mathrm{s}^{-1}$ (see Figure 1). All angles used in this study are measured counterclockwise from the Sun-Earth line as seen from the north.

A useful parameter in the comparison of the model with the observed solar wind parameters is the time, $\tau$, it takes a corotating spiral trajectory, for example that shown in Figure 1, to overtake the Earth. This is given by

$$
\tau=\frac{\varepsilon-\theta_{F}}{13}
$$

where $\varepsilon$ and $\theta$ are in degrees and $\tau$ is in days. $\tau$ is zero for trajectories that pass through the Earth, and negative for trajectories that have corotated past the Earth.

At a heliocentric distance $r$ the frequency (in $\mathrm{kHz}$ ) of the radio emission is $\approx 9 \sqrt{N(r)}$ for emission at the fundamental and $\approx 18 \sqrt{N(r)}$ for emission at the second harmonic (twice the plasma frequency), where $N(r)$ is the electron density in $\mathrm{cm}^{-3}$. We use the electron density distribution empirically derived from the OGO-5 spacecraft studies (Alvarez and Haddock, 1973), rewritten here as

$$
N(r)=N_{E}\left(\frac{214}{r-1}\right)^{2.38} \quad r \gtrsim 3.5 R_{\odot},
$$

where $N_{E}$ is the density at $1 \mathrm{AU}$.

The radio elongations, i.e., the direction of arrival of the emission at different frequencies, are obtained by analyzing the phase of the spin-modulated output of the dipole antenna on the spacecraft, neglecting radio wave propagation effects (Alvarez et al., 1974). Then a search is made for combinations of $N_{E}, \varepsilon, \theta_{F}$ and mode (fundamental or second harmonic) that fit the observed radio elongations. To establish an objective criterion for estimating the quality of the fits a dimensionless quantity is defined. This quantity is a weighted root mean square of the dispersion of the computed elongations, $\lambda_{c, i}$, from the observed ones, $\lambda_{o, i}$, at frequency $i$ :

$$
\Delta=\sqrt{\frac{1}{n} \sum_{r=1}^{n}\left(\frac{\lambda_{o, i}-\lambda_{c, i}}{\delta \lambda_{o, i}}\right)^{2}}
$$

$n$ is the number of frequencies for which the elongation could be measured and $\delta \lambda_{o, i}$ is the standard error of the mean of $\lambda_{o, i}$.

A preliminary graphical fitting gives the mode and the approximate values of $N_{E}$, and $\varepsilon$ and $\theta_{F}$. For each $N_{E}$ a computer program generates a two-dimensional array of $\Delta$-values corresponding to $\varepsilon$ and $\theta_{F}$ entries. Values of $\varepsilon$ and $\theta_{F}$ were changed in steps 
of about $5^{\circ}$. From these arrays the combinations of parameters that have the smallest $\Delta$ were selected. Experience indicates that fits with $\Delta<2$ are good and those with $2<\Delta<3$ are acceptable.

The acceptable models for the radio data are compared with the solar wind data by plotting the best fit parameters $\left(N_{E}\right.$ and $\left.\varepsilon\right)$ and the observed solar wind density and velocity on the same graph as a function of $\tau$. Here we assume that the observed solar wind density and velocity do not change significantly for a period of $\approx 2$ days, so that the spacecraft observations near the Earth can be taken as representative of the corotating spatial structure of the solar wind over approximately $\pm 25^{\circ}$ in azimuth from the Sun-Earth line. The models which best fit the solar wind data are then chosen for comparison with the fast electron data.

The fast electrons are assumed to be ejected from the Sun 8.3 min earlier than the time of onset of the type-III burst at $3.5 \mathrm{MHz}$. The onset time at $1 \mathrm{AU}$ for each electron energy channel is plotted against the reciprocal of the electron velocity. If the electrons at all energies travel approximately the same total path length in reaching $1 \mathrm{AU}$, the onsets at different energies lie on a straight line defined by

$$
\frac{1}{\beta}\left(\frac{L}{c}\right)=t-t_{0},
$$

where $L$ is the total path length, $c$ is the velocity of light, $\beta c$, the electron velocity and $t$ the onset time at $1 \mathrm{AU}$ for that energy and $t_{0}$ the injection time, defined by subtracting $8.3 \mathrm{~min}$ from the time of onset of the type-III burst at $3.5 \mathrm{MHz}$. For all of the events analyzed here this relationship is found to hold within the errors of the measurements.

Two comparisons can be made between the fast electron data and the best fit model for the radio data. First, the observed fast electron energy and onset time at the spacecraft can be compared to the exciter energy and arrival time at $1 \mathrm{AU}$. Close agreement would indicate that the fast electrons are the exciter of the type-III burst and also that the particular model is valid. We note here that the fast electron observations are made at $\tau=0$ while the centroids of the radio source are generally at $\tau \neq 0,|\tau| \lesssim 2$ days. We believe, however, that the comparisons are justified because the electrons are observed to be emitted typically into a cone of interplanetary field lines of $\approx 60^{\circ}$ FWHM, with little variation in electron travel times across this cone (Lin and Anderson, 1967; Lin, 1974). This cone of propagation filled with fast electrons may be largely responsible for the observed large radio source sizes.

Second, the distance $L$, traveled by the electrons can be compared to the smooth spiral distance. If the electrons have traveled closely along the smooth spiral defined by the radio model, without pitch angle scattering but with some true deceleration, then the computed distance $L$ should be less than the smooth spiral distance since the electron velocity at $1 \mathrm{AU}$ will be less than its average velocity along the spiral. We note here that in the absence of pitch angle scattering the electrons will travel along the interplanetary spiral field line with near zero pitch angle regardless of the particle pitch angle close to the Sun because the rapid decrease of the magnetic field strength with distance away from the Sun. 
If the computed path length $L$ is larger than the smooth spiral distance then at least the difference between these two distances must be due to pitch angle scattering.

\subsection{THE 28 FEBRUARY 1972 EVENT}

Figure 2 shows the radio and electron raw data for this event. The radio event is made up of two components, the second of which faded rapidly with decreasing frequency. At $80 \mathrm{kHz}$ the elongation measured near the burst maximum and near the burst tail are different; in this analysis we considered only the value obtained when the burst
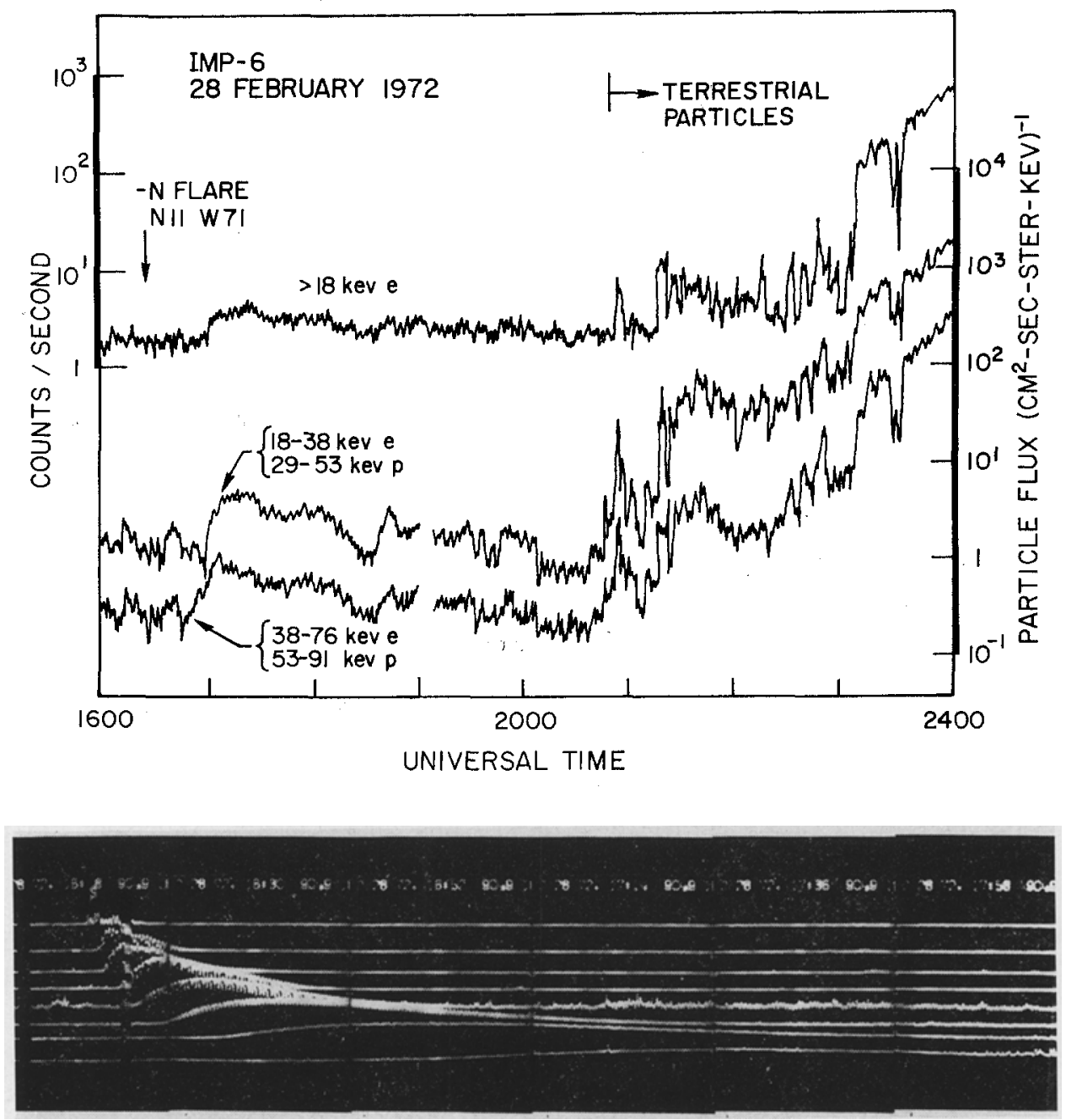

Fig. 2. Electron data (above) and radio data (below) for the 28 February 1972 event. In the radio data the $3.5 \mathrm{MHz}$ channel is at the top; the feature observed simultaneously in all channels corresponds to a radiometer calibration, the amplitude scales are approximately logarithmic and the total length of the picture is about two hours. Time increases from left to right. 
TABLE I

a. Models that fit the elongations of the 28 February, 1972 event

\begin{tabular}{|c|c|c|c|c|c|c|c|}
\hline Model & $\begin{array}{l}N_{E} \\
\left(\mathrm{~cm}^{-3}\right)\end{array}$ & $\begin{array}{l}\varepsilon \\
\text { (deg) }\end{array}$ & $\begin{array}{l}\theta_{F} \\
(\mathrm{deg})\end{array}$ & $F / H$ & $\Delta$ & $\begin{array}{l}\tau \\
\text { (day) }\end{array}$ & $\begin{array}{l}\tau \\
(\mathrm{deg})\end{array}$ \\
\hline 1 & 4.0 & 100 & 75 & All $H$ & 3.0 & 1.9 & 25 \\
\hline 2 & 4.0 & 71 & 57 & All $H$ & 3.1 & 1.1 & 14 \\
\hline 3 & 7.8 & 65 & 50 & All $H$ & 2.0 & 1.2 & 15 \\
\hline $4^{a}$ & 7.8 & 80 & 60 & All $H$ & 1.8 & 1.5 & 20 \\
\hline 5 & 11.1 & 65 & 55 & All $H$ & 2.5 & 0.8 & 10 \\
\hline 6 & 15.8 & 36 & 34 & All $H$ & 2.5 & 0.2 & 2 \\
\hline 7 & 15.8 & 100 & 75 & All $F$ & 3.0 & 1.9 & 25 \\
\hline 8 & 15.8 & 71 & 57 & All $F$ & 3.1 & 1.1 & 14 \\
\hline
\end{tabular}

b. Models that fit the elongations of 27 March, 1972 event

\begin{tabular}{|c|c|c|c|c|c|c|c|}
\hline Model & $\begin{array}{l}N_{E} \\
\left(\mathrm{~cm}^{-3}\right)\end{array}$ & $\begin{array}{l}\varepsilon \\
\text { (deg) }\end{array}$ & $\begin{array}{l}\theta_{F} \\
\text { (deg) }\end{array}$ & $F / H$ & $\Delta$ & $\begin{array}{l}\tau \\
\text { (day) }\end{array}$ & $\begin{array}{l}\tau \\
(\operatorname{deg})\end{array}$ \\
\hline 1 & 7.8 & 115 & 75 & All $\mathbf{H}$ & 2.6 & 3.1 & 50 \\
\hline $2^{a}$ & 11.1 & 63 & 48 & All $\mathbf{H}$ & 2.8 & 1.2 & 15 \\
\hline 3 & 15.8 & 61 & 55 & All $\mathbf{H}$ & 2.0 & 0.5 & 6 \\
\hline 4 & 31.8 & 20 & 25 & All $\mathrm{H}$ & 1.7 & -0.4 & -5 \\
\hline
\end{tabular}

c. Models that fit the elongations of the 16 May, 1971 event

\begin{tabular}{|c|c|c|c|c|c|c|c|}
\hline Model & $\begin{array}{l}N_{E} \\
\left(\mathrm{~cm}^{-3}\right)\end{array}$ & $\begin{array}{l}\varepsilon \\
\text { (deg) }\end{array}$ & $\begin{array}{l}\theta_{F} \\
\text { (deg) }\end{array}$ & $F / H$ & $\Delta$ & $\begin{array}{l}\tau \\
\text { (day) }\end{array}$ & $\begin{array}{l}\tau \\
\text { (deg) }\end{array}$ \\
\hline 1 & 7.8 & 60 & 85 & $0.9 / 0.6$ & 2.3 & -1.9 & -25 \\
\hline 2 & 7.8 & 25 & 50 & $0.9 / 0.6$ & 2.1 & -1.9 & -25 \\
\hline 3 & 7.8 & 70 & 90 & $0.9 / 0.6$ & 2.3 & -1.5 & -20 \\
\hline 4 & 7.8 & 60 & 75 & $0.9 / 0.6$ & 2.3 & -1.2 & -15 \\
\hline 5 & 11.1 & 30 & 40 & $0.9 / 0.6$ & 2.3 & -0.8 & -10 \\
\hline $6^{a}$ & 11.1 & 54 & 59 & $0.9 / 0.6$ & 2.0 & -0.4 & -5 \\
\hline 7 & 15.8 & 60 & 60 & $0.9 / 0.6$ & 2.9 & 0.0 & 0 \\
\hline
\end{tabular}

d. Models that fit the elongations of the 19 August, 1972 event

\begin{tabular}{|c|c|c|c|c|c|c|c|}
\hline Model & $\begin{array}{l}N_{E} \\
\left(\mathrm{~cm}^{-3}\right)\end{array}$ & $\begin{array}{l}\varepsilon \\
\text { (deg) }\end{array}$ & $\begin{array}{l}\theta_{F} \\
\text { (deg) }\end{array}$ & $F / H$ & $\Delta$ & $\begin{array}{l}\tau \\
\text { (day) }\end{array}$ & $\begin{array}{l}\tau \\
(\operatorname{deg})\end{array}$ \\
\hline 1 & 15.8 & 30 & 65 & All $H$ & 2.3 & -2.7 & -35 \\
\hline $2^{a}$ & 15.8 & 50 & 75 & All $\mathrm{H}$ & 2.5 & -1.9 & -25 \\
\hline 3 & 23.4 & 95 & 95 & All $\mathbf{H}$ & 2.5 & 0.0 & 0 \\
\hline 4 & 23.4 & 120 & 120 & All $\mathrm{H}$ & 1.9 & 0.0 & 0 \\
\hline
\end{tabular}

a Indicates best fit model. 
was strong. The onset of the first and second component at $3.5 \mathrm{MHz}$ occurred at $1620.5 \pm 0.5$ and $1623.5 \pm 0.5 \mathrm{UT}$, respectively. An $\mathrm{H} \alpha$ flare of importance $-N$ occurred at N11 W71; it began at 1612 and reached the maximum at 1625 UT. Ground based stations detected type-III bursts in the metric and decametric bands between 1620 and 1625 UT. From the analysis of the data we believe that the first component is better associated with the electron event.

The parameters of the models which fit the radio elongations are shown in the first five columns of Table Ia. The column headed $F / H$ indicates the frequencies between which the mode changes from fundamental to harmonic if there was a mode change.

The solar wind velocity and density are plotted in Figure $3 \mathrm{a}$ as a function of the corotation time $\tau$. The models of Table Ia are plotted on the same plot with each model number indicated beside the density point. Models (3) and (4) agree best with the solar wind data and also are the best fit to the radio elongations. $\theta_{F}$ for model (4) is $60^{\circ}$, close to the associated flare location at W71. Using models (3), (4), and (5) we have computed the radiation arrival time at the Earth for different centroid exciter velocities, $\beta^{\prime}$, and selected the velocity that best fits the observed radio burst onsets. Due to the uncertainties in the measurement of radio onset times the fitted velocities can vary within approximately $10 \%$. These are shown in Table IIa along with the length, $s$, of the smooth spiral between the photosphere and $1 \mathrm{AU}$. The arrival time

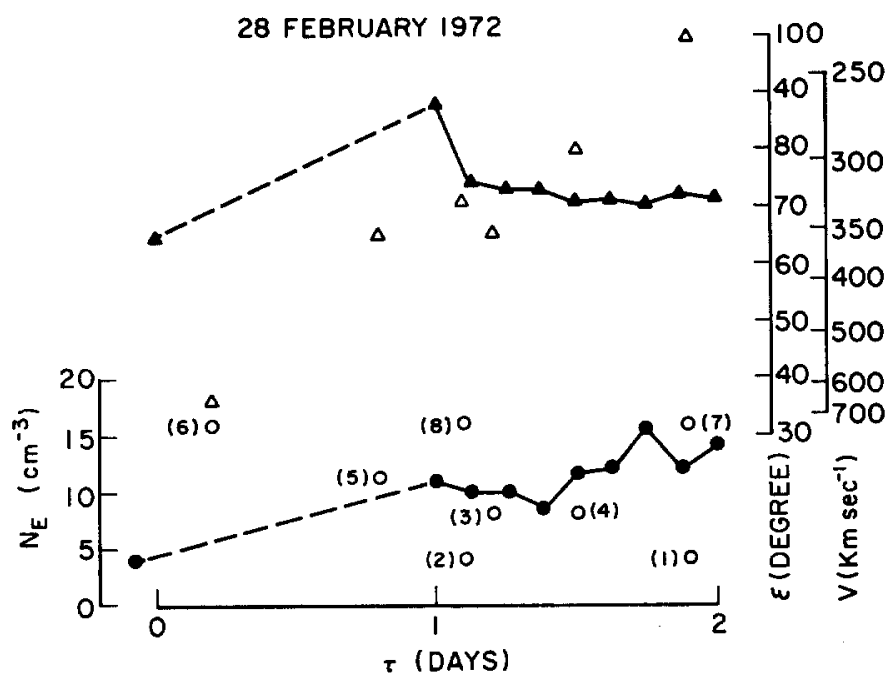

Fig. 3a.

Fig. 3a-b. 28 February 1972 event. (a) Comparison of models with solar wind observations. Circles correspond to electron density and triangles to solar wind velocity, respectively. Filled and opened symbols refer to observations and models, respectively. Dash-lines indicate data gaps. The observations are one-hour averages averaged over three hours. (b) Comparison of energy vs arrival time at the Earth for observed fast solar electron onsets and for exciter particles as predicted by different models. The injection time at the Sun, $t_{0}$, is deduced from the $3.5 \mathrm{MHz}$ radio burst onset. 


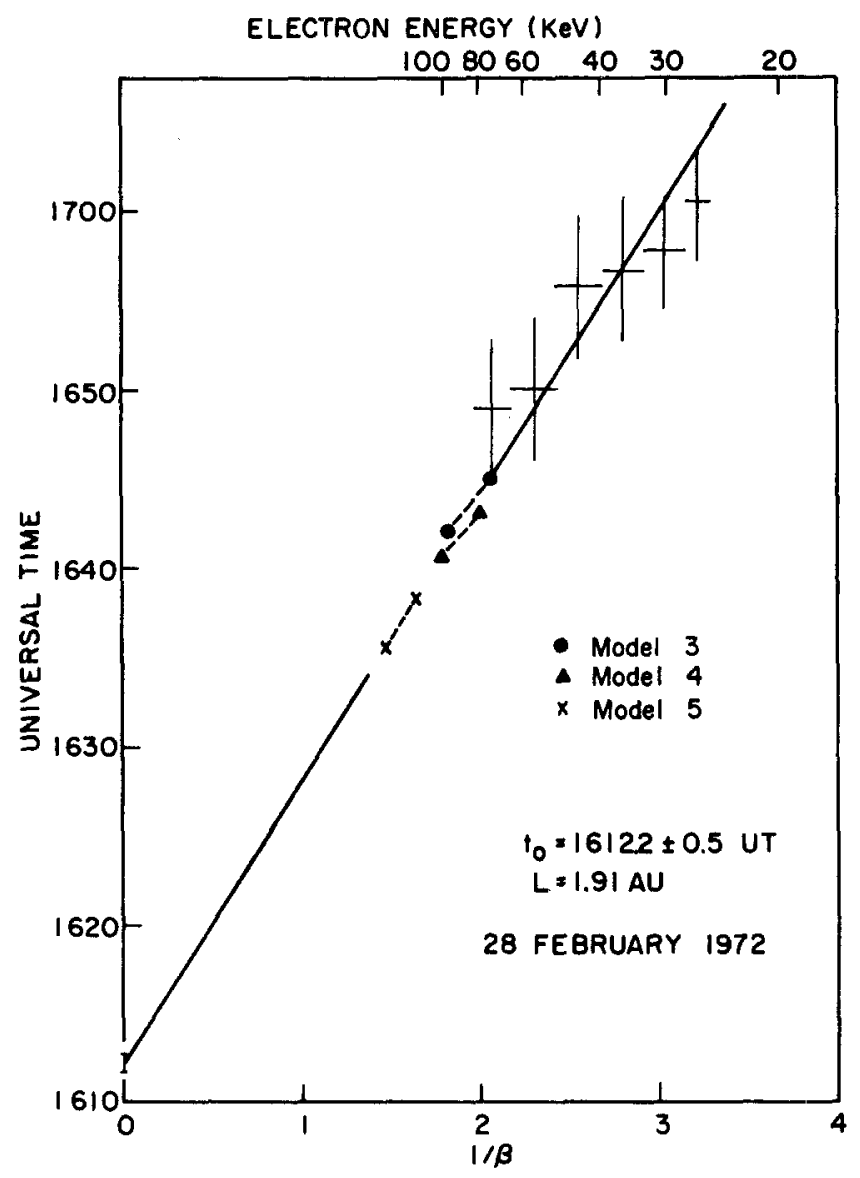

Fig. 3b.

at $1 \mathrm{AU}, t$, can then be computed and compared to the observed fast electron onset times.

The analysis of the fast electron onsets is shown in Figure $3 b$, and yields a path length $L \approx 1.9$ AU compared with $s \approx 1.2 \mathrm{AU}$ for the radio model. If the additional path length above the smooth spiral is indeed due to pitch-angle scattering then the radio velocity $\beta^{\prime}$ should be multiplied by the ratio $L / s$ to compare with the observed fast electrons. The corrected velocity, $\beta=(L / s) \beta^{\prime}$, is used in Figure $3 \mathrm{~b}$. The velocity of the fastest electrons observed above pre-event levels is $\approx 0.55 c$ in close agreement with the corrected velocity computed for models (3) and (4) but not (5).

\subsection{The 27 MARCH 1972 EVENT}

The radio event is composed of only one burst. The onset at $3.5 \mathrm{MHz}$ occurred at $2356.5 \pm 1.0 \mathrm{UT}$. Onset times measured at 230,130 and $80 \mathrm{kHz}$ are uncertain because of Earth noise contamination. The elongation at $80 \mathrm{kHz}$ is not as reliable as those 
TABLE II

Exciter velocity and related quantities

a. 28 February, 1972 event

\begin{tabular}{llllll}
\hline Model & $\beta^{\prime}$ & $s(\mathrm{AU})$ & $L / s$ & $\beta$ & $t(\mathrm{UT})$ \\
\hline 3 & $0.30-0.34$ & 1.18 & 1.61 & $0.48-0.55$ & $1644.9-1641.1$ \\
$4^{\mathrm{a}}$ & $0.33-0.37$ & 1.26 & 1.51 & $0.50-0.56$ & $1643.9-1640.5$ \\
5 & $0.38-0.42$ & 1.18 & 1.61 & $0.61-0.68$ & $1638.0-1635.6$ \\
\hline
\end{tabular}

b. 27-28 March, 1972 event

\begin{tabular}{llllll}
\hline Model & $\beta^{\prime}$ & $s(\mathrm{AU})$ & $L / s$ & $\beta$ & $t(\mathrm{UT})$ \\
\hline 1 & $0.33-0.38$ & 1.48 & 1.37 & $0.45-0.52$ & $0025.5-0020.6$ \\
$2^{\mathrm{a}}$ & $0.30-0.34$ & 1.18 & 1.72 & $0.52-0.58$ & $0020.9-0017.1$ \\
3 & $0.37-0.41$ & 1.17 & 1.73 & $0.64-0.71$ & $0014.5-0011.9$ \\
4 & $0.43-0.50$ & 1.02 & 1.99 & $0.86-0.995$ & $0007.9-0005.2$ \\
\hline
\end{tabular}

c. 16 May, 1971 event

\begin{tabular}{llllll}
\hline Model & $\beta^{\prime}$ & $s(\mathrm{AU})$ & $L / s$ & $\beta$ & $t$ (UT) \\
\hline 4 & $0.28-0.34$ & 1.17 & 1.66 & $0.46-0.56$ & $1309.4-1304.6$ \\
$6^{2}$ & $0.32-0.40$ & 1.14 & 1.70 & $0.54-0.68$ & $1305.6-1259.7$ \\
\hline
\end{tabular}

d. 19 August, 1972 event

\begin{tabular}{llllll}
\hline Model & $\beta^{\prime}$ & $s(\mathrm{AU})$ & $L / s$ & $\beta$ & $t(\mathrm{UT})$ \\
\hline $2^{\mathrm{a}}$ & $0.34-0.35$ & 1.11 & 1.25 & $0.42-0.44$ & $1459.3-1458.0$ \\
\hline
\end{tabular}

a Indicates best fit model.

measured at higher frequencies. At $130 \mathrm{kHz}$ the elongation seems to change during the burst lifetime and we adopted the value deduced from the strongest part of the burst. There was no $\mathrm{H} \alpha$ flare association with this event. However several ground based stations detected type-III bursts in the metric and decametric bands between 2356 and 2359 UT.

The models that fit the radio data are shown in Table $\mathrm{Ib}$. Corotation times range from near zero to +3 days. The comparison of these models with the solar wind observations is shown in Figure 4a. Model (4) disagrees in $\varepsilon$ while $N_{E}$ is very close to a peak. Model (1) agrees in $N_{E}$ but disagrees in $\varepsilon$; the reverse occurs for model (3). Probably only model (2) has a chance to fit better, judging from an interpolation in the data gap. Notice that the solar wind speed was fairly stable while the density changed by a factor of 4 during a 6-hr period only one day before the data gap. 
Table Ib indicates that the smallest $\Delta$ occurs for model (4) which is the densest and shows significant disagreement with solar wind observations. The corresponding corotation is close to zero. In the analysis of other radio events similar situations were found where the combination of high $N_{E}$ and a trajectory passing very close to the Earth gives a fortuitous best fit. This phenomenon may be due to the fact that our technique is not suitable for the geometry or may indicate departure from some of our assumptions; for example, the assumption of a point source to determine the direction of arrival.

The exciter velocities and related quantities for all four models are given in Table IIb. The comparison of the model exciter with the fast electrons is shown in Figure 4b where we have kept models (1) (2) and (3) for illustrative purposes. Electrons with $\beta>0.62(E>140 \mathrm{keV})$ were not detected. Model (2) seems to be the one in best agreement with the fast electron observations.

\subsection{THE 16 MAY, 1971 EVENT}

This radio event was fairly complex. It also was studied by Lin et al. (1973). Three components can be distinctly identified above about $230 \mathrm{kHz}$. The first component, the earliest, is at least two orders of magnitude weaker than the other two and it was not detected at $3.5 \mathrm{MHz}$. At this frequency the onsets of the second and third components occurred at $1237.4 \pm 0.6$ and $1244.3 \pm 0.7 \mathrm{UT}$, respectively. Inspection of the

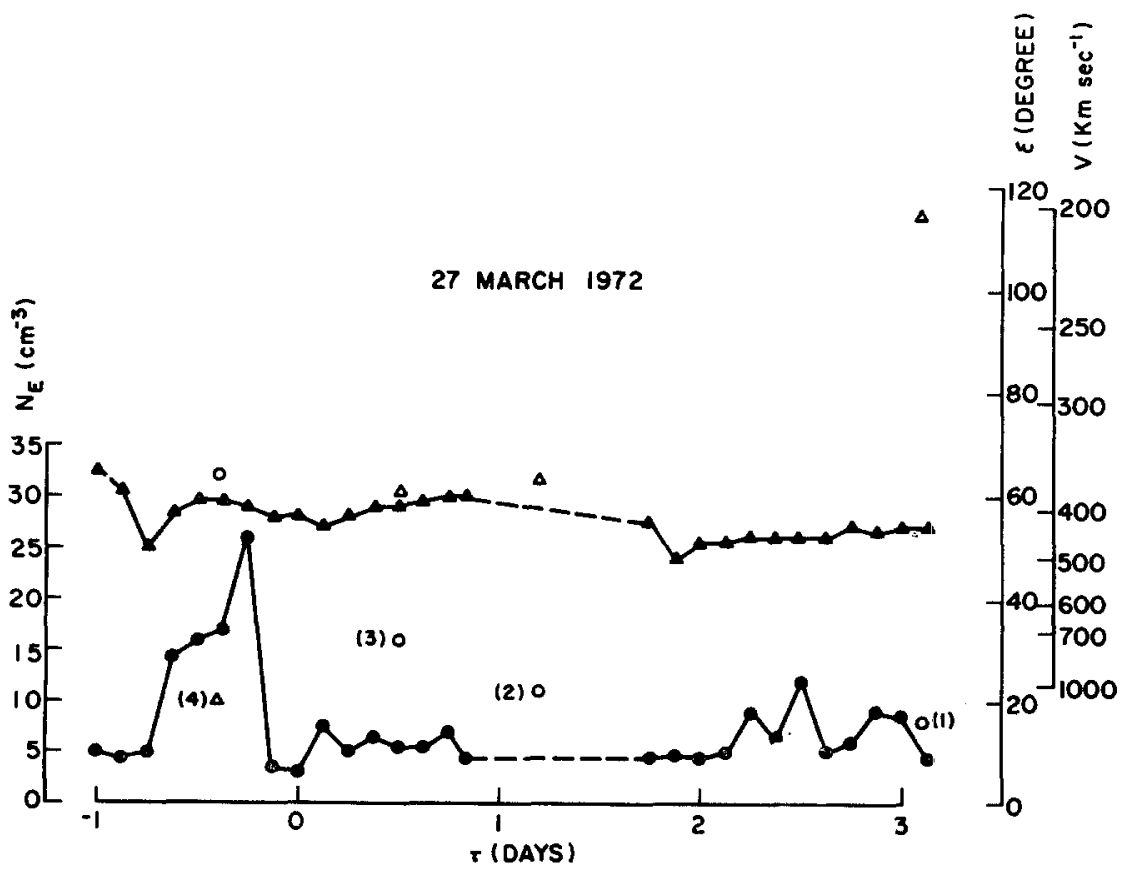

Fig. 4a.

Fig. 4a-b. Same as Figure 3 but for the 27 March 1972 event. 


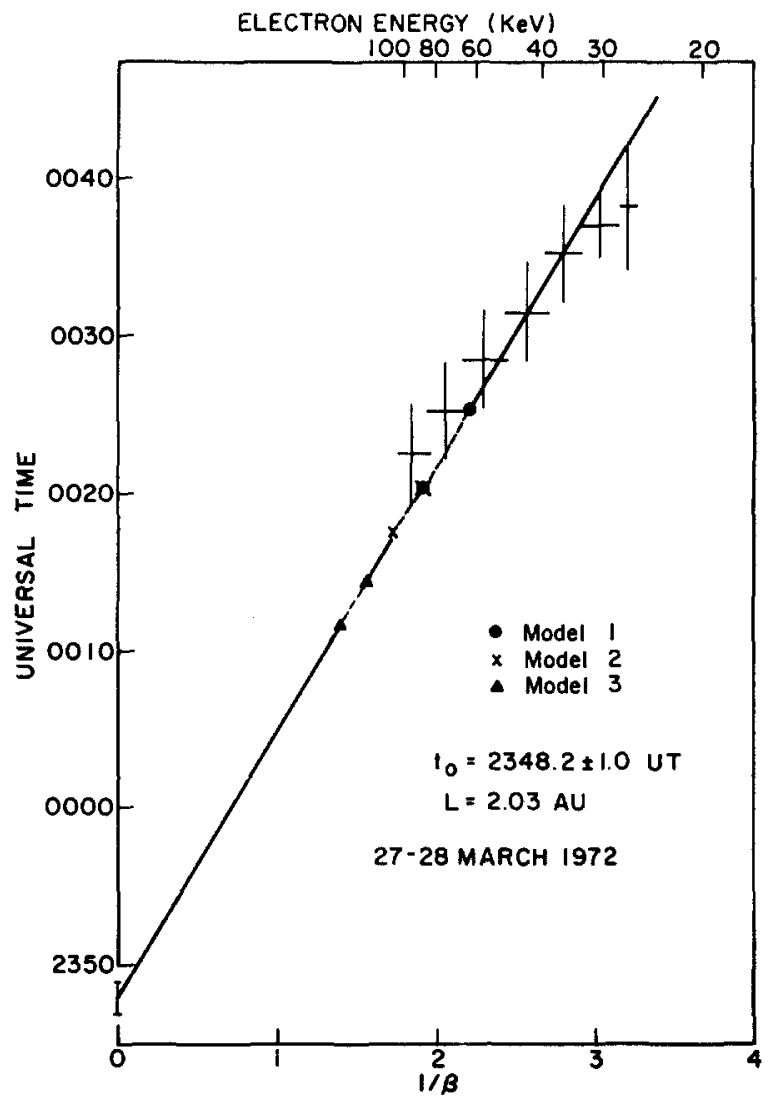

Fig. 4b.

burst profiles suggests that the first component disappeared below $600 \mathrm{kHz}$ and that the second component is very weak at $130 \mathrm{kHz}$. We assume that the third component is the one present at $50 \mathrm{kHz}$.

There was no $\mathrm{H} \alpha$ flare associated with this event. One station detected type-III bursts in the metric band between 1238.5 band 1309.0 UT.

We found many models that fit the observed elongations with $\Delta<3.0$. Several of them have parameters so similar that it was decided to select only some representative models. These are shown in Table Ic. Notice that $\tau<0$ for all models indicating that the trajectory of the burst centroid has corotated past the Earth. The observed radiation changes from fundamental to harmonic between 900 and $600 \mathrm{kHz}$.

The comparison of these models with the solar wind characteristics is shown in Figure 5a. It is seen that models (4) and (6) agree the best with the observations, while models (2) and (5) show significant discrepancies in $\varepsilon$. Models (1) and (3) could be acceptable but were not considered since they are similar to (4). Model (7) could be acceptable but it was disregarded because it places the Earth at the source of the 80 $\mathrm{kHz}$ burst and therefore we should not have seen modulation in the burst profile. 


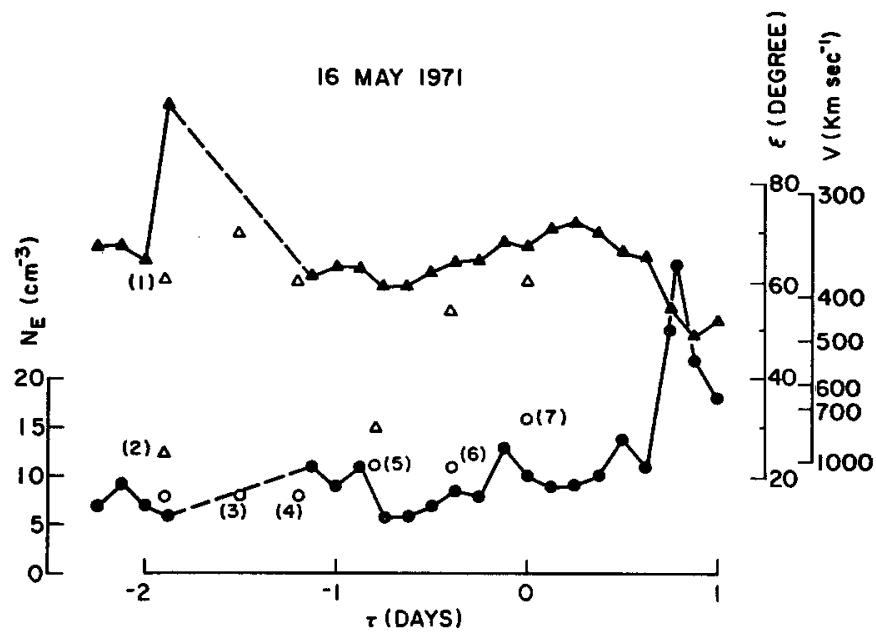

(a)

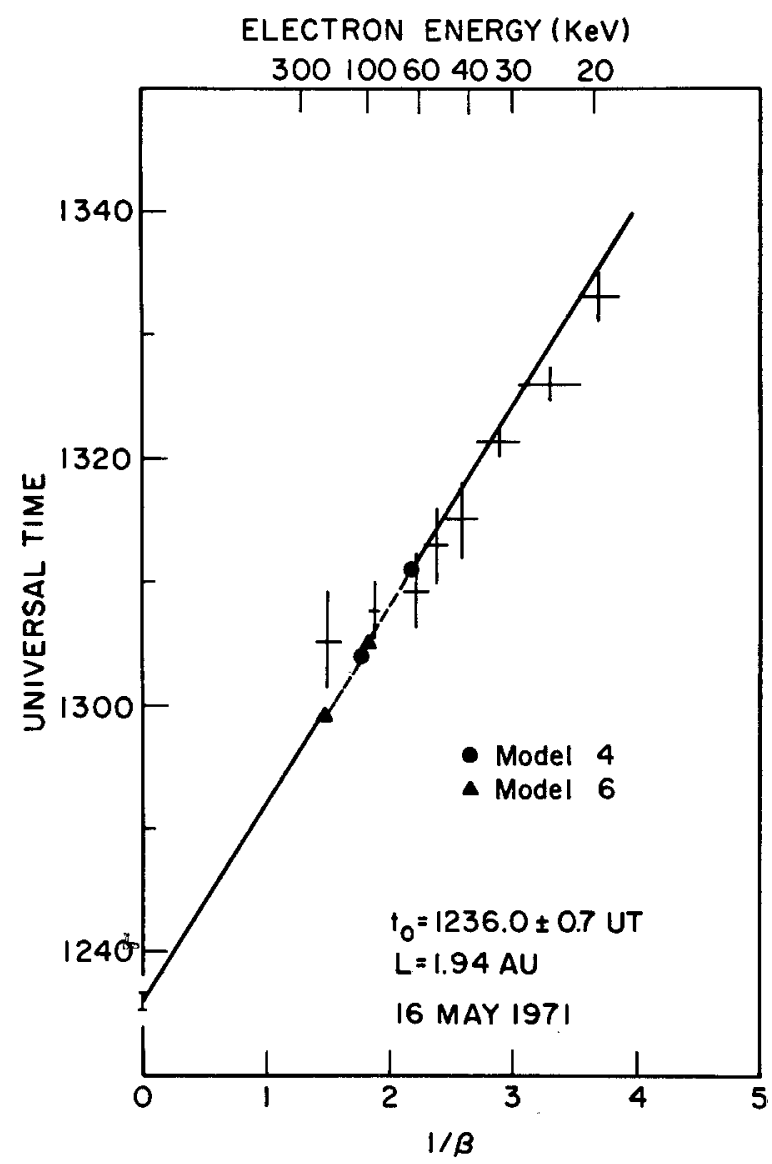

(b)

Fig. 5a-b. Same as Figure 3 but for the 16 May 1971 event. 


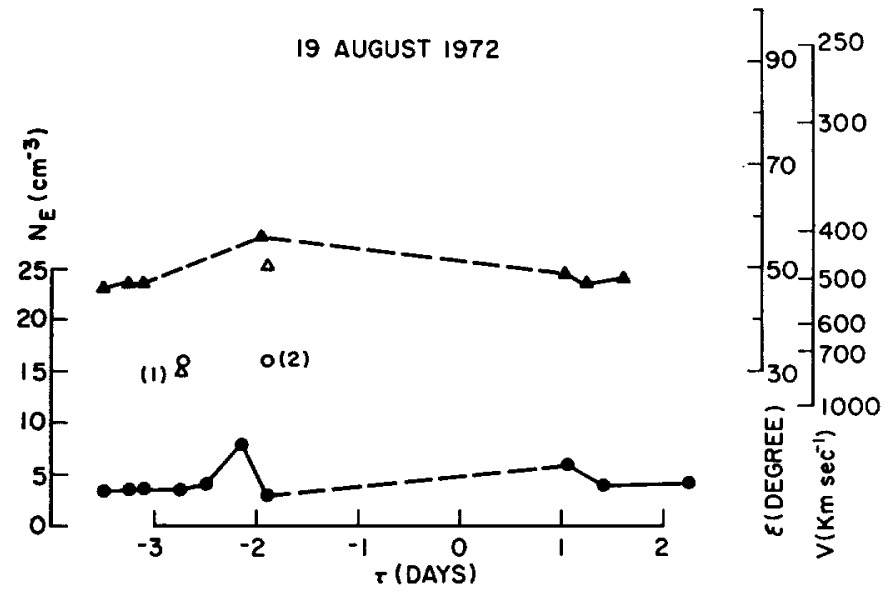

(a)

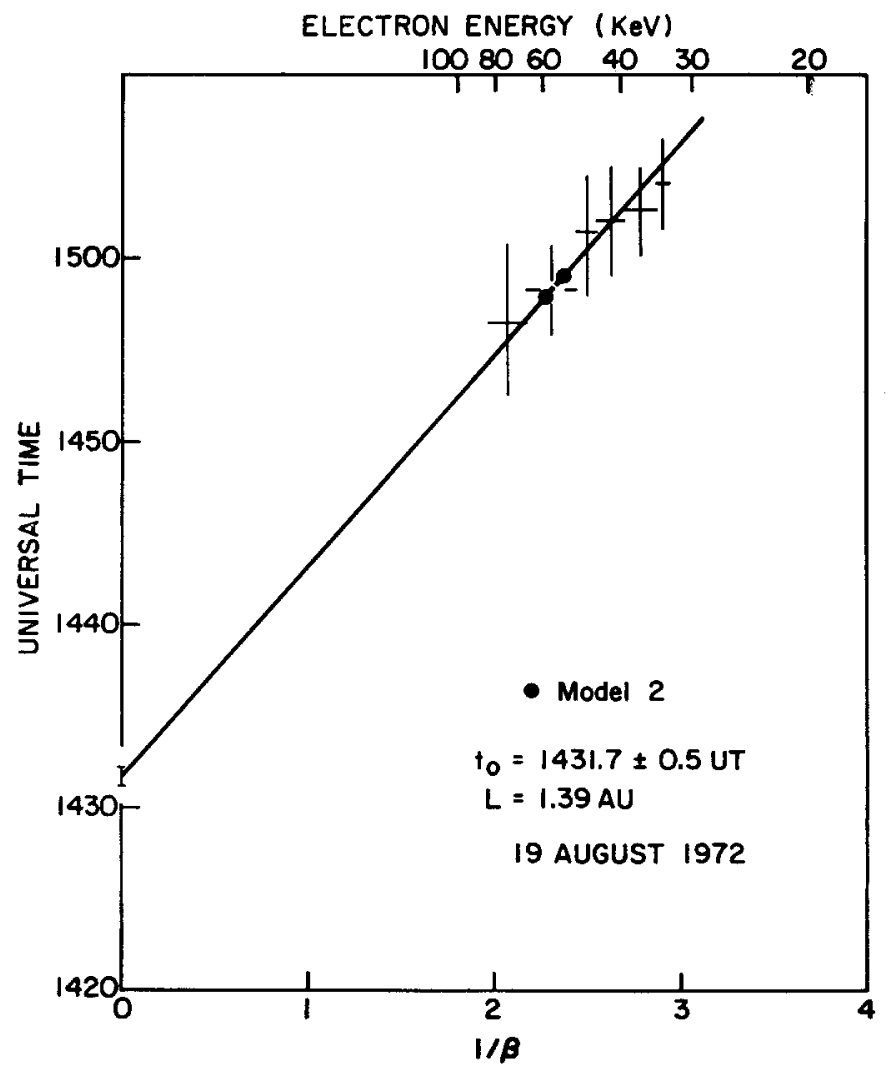

(b)

Fig. 6a-b. Same as Figure 3 but for the 19 August 1972 event. 
The centroid velocities, $\beta^{\prime}$, obtained by fitting models (4) and (6) to the radio onsets are shown in Table IIc. The comparison of the exciter with the observed fast electrons is shown in Figure $5 \mathrm{~b}$. Model (6) agrees better with the assumption that the burst onsets were produced by the fastest observed electrons. Electrons with $\beta>0.7(E>200$ $\mathrm{keV}$ ) were not detected.

\subsection{The 19 AUgust, 1972 EVENT}

The radio event is made up of at least two well-defined components separable at and above $350 \mathrm{kHz}$. The $3.5 \mathrm{MHz}$ onsets occur at $1434.5 \pm 0.5$ and $1440.0 \pm 0.5 \mathrm{UT}$.

An $\mathrm{H} \alpha$ flare of importance 1B occurred at N17 W67. Its onset and maximum occurred at 1432 and 1437 UT, respectively. Several ground based stations reported strong type-III bursts in the metric and decametric band starting at 1434.2 UT.

From the radio data it is difficult to determine whether the first, the second or both components were present below $350 \mathrm{kHz}$. The second component is in better agreement with the electron propagation characteristics and it was used in this study.

The models shown in Table Id fitted the elongations within the criterion adopted, i.e., $\Delta<3$. Notice that models with $N_{E}<15.8 \mathrm{~cm}^{-3}$ do not fit the data. In models (3) and (4) the burst originates behind the west $\operatorname{limb}\left(\theta_{F}>90^{\circ}\right)$. We have several reasons to give low weight to these two models. First, the $3.5 \mathrm{MHz}$ burst was observed, second, there was an $\mathrm{H} \alpha$ flare probably associated with the event and third, the Earth would be immersed in the $80 \mathrm{kHz}$ source (the $40 \mathrm{kHz}$ plasma level is at $226 R_{\odot}$ ), therefore we should not have seen the burst profile modulated.

The IMP-6 solar wind observations were not available for this event and VELA data were used. The comparison of models (1) and (2) with the observations is shown in Figure 6a. The $N_{E}$ for these models ranges between 2 and 5 times the observed values. Unfortunately there is a 3-day data gap. Model (2) gives a trajectory at the edge of the data gap; it gives a solar wind velocity in good agreement with the observations but the density is large. Model (1) disagrees in both $N_{E}$ and $\varepsilon$, and also violates the condition $|\tau|<2$ days. We have accepted model (2). Note that $\theta_{F}=75^{\circ}$ for this model, close to the associated flare position of $67^{\circ}$.

The centroid velocity $\beta^{\prime}$, deduced from component 2 , is shown in Table IId. Related parameters are also shown. The comparison of the exciter with the observed fast electrons is shown in Figure 6b. Electrons with $\beta>0.55(E>100 \mathrm{keV})$ were not detected.

\section{Summary and Discussion}

The solar wind velocity and density, together with the radio burst elongations, determine the trajectory of the centroid of the burst exciter as it traveled from the Sun to $1 \mathrm{AU}$. Comparison of the solar wind and radio data show that the radio emission is excited mostly at the second harmonic. Using the trajectory, we determined a small range of exciter velocity consistent with the radio bursts' onsets. A comparison of the spiral distances, $s$, traveled by the radio burst exciters with the distances, $L$, traveled by the fast electrons observed at $1 \mathrm{AU}$ indicates that typically 
$1.3<L / s<1.7$, implying that substantial scattering of the electrons must have occurred in the interplanetary medium. This picture is further supported by the temporal profiles of the fast electron flux. For the three events with large values of $L / s$ (May 16, 1971 ; February 28, 1972; March 27, 1972) the flux shows the rapid raise-slow decay profile characteristic of an impulsive injection at the Sun followed by substantial scattering in the interplanetary medium. The August 19, 1971 event has a rapid riserapid decay profile characteristic of relatively scatter-free event (Lin, 1970). For this event the ratio of the electron path length to the smooth spiral distance, $L / s$, is much smaller, consistent with the relatively scatter-free propagation in the interplanetary medium. In a simple model including scattering we assume that the total path length and velocity of individual particles in the stream can be obtained by increasing the path length and the velocity of the centroid by the factor $L / s$. This results in an excellent agreement between the characteristics of the exciter particles responsible for the burst onsets and those of the fastest electrons observed at $1 \mathrm{AU}$ : they arrive at $1 \mathrm{AU}$ simultaneously and with the same velocity.

In addition, detailed analysis of the motion of type-III burst radio sources (Alvarez and Lin, 1975) shows that the scattering increases with increasing heliocentric distance in a manner consistent with the spatial distribution of density irregularities inferred from radio scintillation studies.

The overall picture of type-III bursts is thus consistent with negligible true deceleration of the fast electrons as they travel from the Sun to the orbit of the Earth, although we cannot completely rule out the possibility that the electrons started out with much higher velocities at the Sun and were decelerated and scattered as they traveled outward. However, estimates of the fast electron velocities close to the Sun, derived from both hard X-ray (Datlowe and Lin, 1974) and type-III radio observations, indicate that the electrons at the Sun were not substantially more energetic than those observed at $1 \mathrm{AU}$.

\section{Acknowledgements}

Plasma data from IMP-6 were obtained in a program under the auspices of the U.S. Atomic Energy Commission. Other plasma data were obtained in the Vela Satellite Program jointly sponsored by the U.S. Department of Defense and the A.E.C. and managed by the U.S. Air Force.

One of us (R.P.L.) wishes to acknowledge the support of Dr Kinsey Anderson and NASA Contract NAS5-11038 and Grant NGL05-003-017.

One of us (H.A.) thanks Prof. Fred T. Haddock for his support; Dr Margo F. Aller for helping in the data analysis and for entertaining helpful discussions; and $\mathrm{Mr}$ William H. Potter for developing the $\Delta$-matrix analysis, for comments on an early draft and for many discussions. The radio work was done under NASA Grant NSG 7004.

\section{References}

Alvarez, H. and Haddock, F. T.: 1973, Solar Phys. 29, 197.

Alvarez, H., Haddock, F. T., and Lin, R. P.: 1972, Solar Phys. 26, 468. 
Alvarez, H., Haddock, F. T., and Potter, W. H.: 1974, Solar Phys. 34, 413.

Alvarez, H. and Lin, R. P.: 1975, paper presented at the A.p.S. Solar Phys. Div. Meeting, Boulder,. Colo.

Bame, S. J., Asbridge, J. R., Felthauser, H. E., Hones, E. W., and Strong, I. B. : 1967, J. Geophys. Res. 72, 113.

Datlowe, D. and Lin, R. P.: 1973, Solar Phys. 32, 45.

Fainberg, J., Evans, L. G., and Stone, R. G.: 1972, Science 178, 743.

Feldman, W. C., Asbridge, J. R., Bame, S. J., and Montgomery, M. D.: 1973, J. Geophys. Res. 78, 2017.

Frank, L. A. and Gurnett, D. A.: 1972, Solar Phys. 27, 446.

Lin, R. P.: 1970, J. Geophys. Res. 75, 2583.

Lin, R. P.: 1974, Space Sci. Rev. 16, 189.

Lin, R. P. and Anderson, K. A.: 1967, Solar Phys. 1, 446.

Lin, R. P., Evans, L. G., and Fainberg, J.: 1973, Astrophys. Letters 14, 191.

McCracken, K. G. and Rao, U. R.: 1970, Space Sci. Rev. 4, 155.

Smith, D. F.: 1974, Space Sci. Rev. 16, 91.

Wild, J. P.: 1950, Astron. J. Sci. Res. A3, 399. 